\title{
A novel cancer stem cell-based classification model for the tumorigenesis and development of colorectal cancer
}

\author{
Zhaopeng Yan ${ }^{1}$, Rui Wang ${ }^{2}$, Qi Su ${ }^{1}$ \\ ${ }^{1}$ Department of General Surgery, ${ }^{2}$ Department of Critical Medicine, Shengjing Hospital, China Medical University, Shenyang 110004, China \\ Correspondence to: Qi Su. Department of General Surgery, Shengjing Hospital, China Medical University, 36 Sanhao Street, Heping District, \\ Shenyang 110004, China. Email: suqi100@hotmail.com.
}

\begin{abstract}
Cancer is a heterogenetic disease. Although multiple hypotheses and models have been indicated, there is still no robust method to distinguish the heterogeneity of cancer. Evidence has been provided showing that cancer stem cells (CSCs) play an important role in the origin and progression of cancer. In this opinion article, based on the theory of CSCs, we propose a novel CSC-based classification model (CSCCM) to explain the origin and progression of colorectal cancer, which would explain some confusing clinical phenomena and distinguish the heterogeneity of colorectal cancer from its origin. We further suggest that this model can presently be incorporated into the cancer progression theory.
\end{abstract}

Keywords: Colorectal neoplasms; cancer stem cell (CSC); cell differentiation; cancer progression; tumorigenesis

Submitted Jun 05, 2019. Accepted for publication Oct 10, 2019.

doi: $10.21037 /$ tcr.2019.10.19

View this article at: http://dx.doi.org/10.21037/tcr.2019.10.19

A malignant tumor is a kind of genome disorder. Although the medical techniques for tumor treatments have made great progress, malignant tumors are still one of the most common reasons for death worldwide. There is no theory or model that can perfectly explain all the clinical phenomena related to tumorigenesis and tumor progression.

The cancer stem cell (CSC) theory was first proposed over forty years ago. The standard CSC model posits that only a few cancer cells have the ability to proliferate continually; the ones that can are considered CSCs, while other cancer cells lack the ability to proliferate continually or can only transiently proliferate. CSCs are also chemotherapy-resistant. CSCs have been reported to be found in colorectal cancers, brain cancers, and breast cancers (1-4). On the one hand, the theory of CSCs could explain some phenomena such as tumor dormancy or metastasis. On the other hand, it is still confusing that not every cancer adheres to the CSC model. In this opinion article, we propose a "CSC-based classification model" (CSCCM) to clarify the origin, progression, and clinical significance of CRC and describe how this model can explain clinical phenomena otherwise unaccounted for by other pre-existing theories or models.

\section{CSCCM of CRC}

It is generally understood that for most cancers, CSCs may not come from normal stem cells and may instead be de-differentiated cancer cells. Colorectal cancer (CRC) is the fourth most commonly diagnosed cancer in the world and is a major cause of tumor-related death worldwide (5). In colorectal cancer, there are two theories to explain where CSCs come from. One opinion is that stem cells of colorectal mucosa transform into CSCs and initiate CRC. The other opinion is that non-stem cancer cells appear first, and then some of the non-stem cancer cells de-differentiate and transform into CSCs.

Base on the opinions mentioned above, we propose a CSCCM of CRC and conclude that there should be four subtypes of colorectal cancer:

* Type 1: the epithelial cells of colorectal mucosa give rise to non-stem cancer cells, with no dedifferentiation occurring. The composition of the type 1 tumor is non-stem cancer cells.

* Type 2: the epithelial cells of colorectal mucosa give rise to non-stem cancer cells, with non-stem cancer cells de-differentiating and transforming into CSCs. 
The composition of the type 2 tumor is mostly nonstem cancer cells and a small portion of CSCs.

* Type 3: the epithelial cells of colorectal mucosa give rise to cancer cells (group 1), with non-stem cancer cells de-differentiating and transforming into CSCs. The CSCs then accumulate novel mutations and differentiate into non-stem cancer cells (group 2). The group 1 cancer cells and group 2 cancer cells are totally different colorectal cancer cells with different biological behavior. For example, adenosquamous carcinoma of CRC has both adenocarcinoma (group 1) and squamous cancer cells (group 2), and usually squamous cancer cells (group 2) are more aggressive compared with adenocarcinoma ones (group 1) (6). The composition of the type 3 tumor is group 1 cancer cells, CSCs, and group 2 cancer cells.

* Type 4: the tumor is directly originated from colorectal stem cells. The composition of type 4 tumor is mostly CSCs and a small portion of nonstem cancer cells.

\section{Model-based four types of CRC versus consensus molecular subtypes (CMS) of CRC}

The consensus molecular subtypes (CMS) is a gene expression-based subtyping. According to CMS, CRC can be divided into four subtypes with distinguishing features: CMS1, hypermutated, microsatellite unstable, strong immune activation, least stemness; CMS2, epithelial, chromosomal instability, marked WNT and MYC signaling activation, moderate stemness, epithelial; CMS3, moderate stemness, epithelial, metabolic dysregulation, prominent KRAS mutation; and CMS4, prominent transforming growth factor $\beta$ activation, stromal invasion, angiogenesis, stemness and epithelial-mesenchymal transition (EMT) activation (7). In the CMS model, the overall survival of each subtype is CMS1 $=$ CMS2 $=$ CMS3 $>$ CMS4, and relapse-free survival of each subtype is $\mathrm{CMS} 1=\mathrm{CMS} 2=$ CMS3 > CMS4.

Interestingly, when we compared the CSCCM with CMS, we found some corresponding relationships between them. According to our model, Type 1 should have the best prognosis and least stemness, which is in accordance with CMS1 (least stemness). Type 2 should contain a few CSCs, which is in accordance with CMS2 (moderate stemness). Type 3 should contain CSCs and at least two types of non-stem cancer cells (group 1 and group 2, as mentioned previously), which is in accordance with CMS3 (moderate stemness, metabolic dysregulation). Type 4 should have the highest metastasis potential and the worst prognosis due to the stemness of the bulk of the tumor, which is in accordance with CMS4 (most stemness).

\section{Early metastasis or no metastasis in a large tumor burden}

Usually, the larger the tumor is, the greater the tumor burden, and the higher the possibility that local or distal metastasis can happen. However, in clinical practice, although rare, we can see that metastasis happens in an early stage in CRC or there is no metastasis in a $\mathrm{T} 4 \mathrm{~b}$ CRC. According to this model, one explanation of this phenomenon is that in Type 4 CRCs, cancer originates from CSCs, and even in an early stage, the CSCs have the ability to migrate and cause metastasis, while in Type 1 CRCs, there are no CSCs, which indicates the possibility that no metastasis can occur even with a great tumor burden.

\section{Pathological complete resolve (PCR) in CRC after neoadjuvant chemotherapy}

Neoadjuvant chemotherapy now is recommended in middle and low rectal cancer. The effect of neoadjuvant chemotherapy varies. PCR after neoadjuvant chemotherapy can be observed in some cases, while in others, it is not. There are no predictive factors for chemotherapy sensitivity. As known CSCs are resistant to chemotherapy, and, according to this classification model, to Type 1 CRC, the tumor is composed of non-CSC cancer cells and can respond well to chemotherapy and be resolved completely. In other types of CRC which the CSCs have aroused, the tumor is composed of both CSCs and non-CSC cancer cells. Chemotherapy kills the non-CSC cancer cells and makes the tumor shrink temporarily; the dying of the nonCSC cancer cells release space for CSCs, which make the shrinking tumor rebound and progress quickly.

\section{The dormancy of cancer cells}

Cancer cells can change into a status of dormancy, but when triggered by certain factors, the dormant cancer cells can reactivate. After reactivation, most of the cancer cells die due to being maladapted to the microenvironment, but sometimes a few survive and initiate proliferation to cause 
cancer recurrence. It is not clear why some dormant cancer cells survive while others do not. According to this model, some of the undifferentiated dormant cancer cells transform into CSCs, and then the CSCs differentiate into a new kind of cancer cells (group 2 in Type 3), which are more adapted to the microenvironment and survive to form recurrent lesions.

In this perspective article, we propose a CSCCM to explain the origin and progression of CRC. The model is intended to provide a complete framework to help direct future CRC research and clinical practice.

\section{Acknowledgments}

Funding: None.

\section{Footnote}

Conflicts of Interest: All authors have completed the ICMJE uniform disclosure form (available at http://dx.doi. org/10.21037/tcr.2019.10.19). The authors have no conflicts of interest to declare.

Ethical Statement: The authors are accountable in all respects for ensuring that questions related to the accuracy or integrity of any part of the work are appropriately investigated and resolved.

Open Access Statement: This is an Open Access article distributed in accordance with the Creative Commons Attribution-NonCommercial-NoDerivs 4.0 International
License (CC BY-NC-ND 4.0), which permits the noncommercial replication and distribution of the article with the strict proviso that no changes or edits are made and the original work is properly cited (including links to both the formal publication through the relevant DOI and the license). See: https://creativecommons.org/licenses/by-nc-nd/4.0/.

\section{References}

1. Al-Hajj M, Wicha MS, Benito-Hernandez A, et al. Prospective identification of tumorigenic breast cancer cells. Proc Natl Acad Sci U S A 2003;100:3983-8.

2. Ricci-Vitiani L, Lombardi DG, Pilozzi E, et al. Identification and expansion of human colon-cancerinitiating cells. Nature 2007;445:111-5.

3. Singh SK, Hawkins C, Clarke ID, et al. Identification of human brain tumour initiating cells. Nature 2004;432:396-401.

4. O'Brien CA, Pollett A, Gallinger S, et al. A human colon cancer cell capable of initiating tumour growth in immunodeficient mice. Nature 2007;445:106-10.

5. Bray F, Ferlay J, Soerjomataram I, et al. Global cancer statistics 2018: GLOBOCAN estimates of incidence and mortality worldwide for 36 cancers in 185 countries. CA Cancer J Clin 2018;68:394-424.

6. O'Brien CA, Pollett A, Gallinger S, et al. A human colon cancer cell capable of initiating tumour growth in immunodeficient mice. Nature 2007;445:106-10.

7. Guinney J, Dienstmann R, Wang X, et al. The consensus molecular subtypes of colorectal cancer. Nat Med 2015;21:1350-6.
Cite this article as: Yan Z, Wang R, Su Q. A novel cancer stem cell-based classification model for the tumorigenesis and development of colorectal cancer. Transl Cancer Res 2019;8(7):2621-2623. doi: 10.21037/tcr.2019.10.19 